\title{
The Convergence and Partial Convergence of Alternating Series
}

\author{
By J. R. Philip*
}

\begin{abstract}
The alternating series is $\Sigma_{n=1}^{\infty} j_{n} f(n)=[j] f$, with $f$ a single-signed monotonic function of the real variable $x$. The $j_{n}$ are \pm 1 , their sign fixed by repetition of the 'template' $[j]$ of finite length $2 p$. $[j]$ constitutes a difference scheme of 'differential order' $D$, which can be determined. The principal theorem is that $[j] f$ is 'partially convergent' if and only if $\lim _{x \rightarrow \infty} f^{(D-1)}(x)$ is bounded. A series is partially convergent when the limit as $M \rightarrow \infty$ of the sum of $2 p M$ terms exists. For [ $j]$ 'pure', the improved Euler-Maclaurin expansion (IEM) gives the compact representation
\end{abstract}

(A) $\quad s^{(p)} \sim-\frac{\mu(D)}{2 p} \sum_{r=0}^{\infty}(2 p)^{2 r} \frac{B_{2 r}(1 / 2)}{(2 r !)} f^{(2 r+D-1)}\left(\theta_{r}\right), \quad 1-p \leqslant \theta_{r} \leqslant p$.

$S^{(p)}$ is the sum, $\mu(D)$ is the $D$ th 'template moment', and the $B_{2 r}$ are Bernoulli numbers. Efficient means for practical summation of these series follow also from IEM. In illustration, 10 alternating series with $D$ ranging from 1 to 3 are summed using IEM. It is found that the leading term of (A) with $\theta_{0}=1 / 2$ gives a simple but effective estimate of sums. The paper also gives a comparison with Euler's transformation in the case $p=1$ and discusses sums to $N$ terms with $N / 2 p$ nonintegral and finite but large.

1. Introduction. Perception of the sum of the series $\Sigma_{n=1}^{N} u(n)$ as an approximation to an integral of $u(x)$ goes back at least to Euler and Maclaurin. The present study is based on the further perception of alternating series of various levels of complication as approximations to integrals not of $u(x)$, but of linear combinations of appropriate derivatives of $|u(x)|$. From this viewpoint we examine the convergence, and the practical arithmetic summation, of various alternating series.

In what follows $m, M, n, N, p, q, w, W$, all denote positive integers; $m$ and $n$ run through $1,2, \ldots$, to $M$ and $N$, respectively; $M$ and $N$ may be finite or infinite; $p$ and $W$ are finite; $q$ runs through the values $1,2, \ldots, 2 p ; w$ may assume the values $1,2, \ldots, W$. The integer $D$ may assume the values $0,1,2, \ldots$, but may not exceed $1+\log _{2} p$. The integer $k$ may assume the values $0,1,2, \ldots$, but may not exceed $1+\log _{2} p-D$. The integer $i$ runs through the values $0,1, \ldots, k$. The integer $g$ may assume any finite value $0,1,2, \ldots$. The integer $r$ may assume the values $0,1,2, \ldots, \infty$.

Received August 24, 1979.

1980 Mathematics Subject Classification. Primary 40A05, 65B15, 65B10, 40 D05.

Key words and phrases. Alternating series, convergence, practical summation.

* Contribution from Department of Agronomy, Cornell University, Ithaca, New York 14853; Agronomy Paper \#1319. This work was supported in part by NSF grant ENG-77-17004. 
2. The Template of an Alternating Series. We are concerned with alternating series of the form $\Sigma_{n=1}^{N} j_{n} f(n)$, where $f(n)$ is a single-signed function of the real variable $x$. Without loss of generality we take $f(x)$ positive, at least in the relevant range of $x$. All $j_{n}$ are either +1 or -1 , their signs being established through continued repetition of the template $[j]$, which specifies the sign of the first $2 p$ terms. We require that a template of length $2 p$ contain just $p$ +'s and $p$-'s. Both the template and the associated series will be said to be of degree $p$. Examples of templates of degrees 1 to 4 are given in Table 1. Evidently, $\Sigma_{q=1}^{2 p} j_{q} \equiv 0$ and $\left|\Sigma_{n=1}^{N} j_{n}\right| \leqslant p$ for all $N$.

TABLE 1

Some templates and their properties

\begin{tabular}{ccc}
\hline Template & Degree & Order \\
{$[j]$} & $p$ & $D$ \\
+- & 1 & 1 \\
+-++ & 2 & 2 \\
+-++-+ & 3 & 1 \\
$++-\ldots++$ & 3 & 1 \\
+-+-+++ & 4 & 1 \\
++--+-++ & 4 & 1 \\
+-++-++- & 4 & 2 \\
\hline
\end{tabular}

We use the notation

$$
S(j, f(x), N)=\sum_{n=1}^{N} j_{n} f(n)=\sum_{n=1}^{N} u(n) .
$$

The sum of the first 87 terms of $2^{1 / 2}-3^{1 / 2}-4^{1 / 2}+5^{1 / 2}+6^{1 / 2}-7^{1 / 2}-8^{1 / 2}+\ldots$ is thus written $S\left(+--+,(x+1)^{1 / 2}, 87\right)$. Template representation of a series is not unique. For example,

$$
S\left(+--+,(x+1)^{1 / 2}, 87\right)=2^{1 / 2}-S\left(++--,(x+2)^{1 / 2}, 86\right) \text {. }
$$

When the sum exists as $N \rightarrow \infty$, we omit $N$ and write simply $S(j, f(x))$. We shall omit $j$ and/or $f(x)$ from the notation, when we may do so without ambiguity. plate,

We observe that, since the $j_{n}$ are generated by continued repetition of the tem-

$$
S(2 p M)=\sum_{m=1}^{M} \sum_{q=1}^{2 p} j_{q} f(2 p(m-1)+q) .
$$


In later sections we shall employ the more concise notation

$$
[j] f=S(j, f(x))=\sum_{n=1}^{\infty} j_{n} f(n)
$$

3. Convergence, Partial Convergence, and Divergence. For certain alternating series $\lim _{M \rightarrow \infty} S(2 p M+q-1)$ exists for at least one value of $q$ in $1<q<2 p$. We shall call such series partially convergent.

THEOREM 3.1. A partially convergent series is convergent if and only if $\lim _{x \rightarrow \infty} f(x)=0$.

In this case, and in this case only, $\lim _{M \rightarrow \infty} S(2 p M+q-1)$ exists for all $q$ in $1 \leqslant q \leqslant 2 p$ and is independent of $q$.

We thus employ the term 'convergent' in its classical sense. Our use of the term 'divergent' is, however, nonclassical. We describe as divergent series which are neither convergent nor partially convergent; i.e. for which $\lim _{M \rightarrow \infty} S(2 p M+q-1)$ does not exist for any $q$.

4. The Differential Order of a Template. Partial convergence of the series $[j] f$ evidently depends on properties of both the template $[j]$ and the function $f(x)$. The relevant property of the template is its differential order.

A template is a (generally rather inefficient) finite-difference scheme. It follows from the mean value theorem that the sum $S(2 p)$ can be represented as a linear combination of derivatives of $f$ thus:

$$
\begin{aligned}
S(2 p)= & \sum_{q=1}^{2 p} j_{q} f(q)=\sum_{i=0}^{k} \alpha_{i} f^{(D+i)}\left(\theta_{i}\right), \\
& 1 \leqslant D \leqslant D+k \leqslant 1+\log _{2} p, \alpha_{i}=\text { finite constant, } 1 \leqslant \theta_{i} \leqslant 2 p .
\end{aligned}
$$

The representation (2) is not necessarily unique. On the other hand, the differential order of a given template, $D$, is uniquely determined by its properties of symmetry and antisymmetry.

We formalize the definition and evaluation of $D$ by introducing the gth template moment, $\mu(g)$, through the equation

$$
\mu(g)=\sum_{q=1}^{2 p} j_{q}(q-p-1 / 2)^{g} .
$$

We then define the differential order (order, for short) of a template, $D$, as the smallest value of $g$ for which $\mu(g) \neq 0$. The order of selected templates of degrees 1 to 3 is shown in Table 1 .

We note that applying (2) to (1) gives the representation

$$
S(2 p M)=\sum_{m=1}^{M} \sum_{i=0}^{k} \alpha_{i} f^{(D+i)}\left(2 p m-\theta_{i, m}\right), \quad 0 \leqslant \theta_{i, m} \leqslant 2 p-1 .
$$

Reverting to (2), we introduce the concept of a pure template. We say that 
a template is pure when a representation (2) exists with $k=0$, i.e. with

$$
S(2 p)=\sum_{q=1}^{2 p} j_{q} f(q)=\alpha_{0} f^{(D)}\left(\theta_{0}\right) .
$$

Six examples of pure templates will be found in Table 4 .

The second equality of (5) enables us to evaluate $\alpha_{0}$ for pure templates. We replace each $f(q)$ and also $f^{(D)}\left(\theta_{0}\right)$ by its Taylor series about $x=p+1 / 2$. Equating coefficients of $f^{(D)}(p+1 / 2)$ in the two resulting expansions gives

$$
\alpha_{0}=\mu(D) / D !
$$

so that (5) becomes

$$
S(2 p)=\frac{\mu(D)}{D !} f^{(D)}\left(\theta_{0}\right)
$$

5. The Monotonic Function $f(x)$. We require here that $f$ and all its derivatives $f^{(r)}$ be finite monotonic functions of $x$, at least in $1 \leqslant x<\infty . f$ is positive and each $f^{(r)}$ is necessarily single-signed. We require further that all decreasing $f^{(r)}$ decrease to zero, and that all negative increasing $f^{(r)}$ increase to zero. The class of functions we admit includes the nondecreasing polynomial of degree $t(t \geqslant 0)$, so that we take 'monotonic' to embrace the cases $f^{(t)}=$ constant $=\beta>0$ and $f^{(r)}=0$ for $r \geqslant t+1$. In computations later in the paper we need $f^{(r)}$ finite in $1-p \leqslant x<\infty$. We may always ensure this without loss of inherent generality by, if need be, discarding leading terms of a given series and adjusting the origin of $x$.

6. The Alternating Series of Degree $p$ and $\operatorname{Order} D$. We investigate the series [j] $f$, with [j] of degree $p$ and order $D$. We may rewrite (4) as

$$
\begin{aligned}
& S(2 p M)=\sum_{i=0}^{k} \alpha_{i} S_{i}(2 p M), \\
& S_{i}(2 p M)=\sum_{m=1}^{M} f^{(D+i)}\left(2 p m-\theta_{i, m}\right) .
\end{aligned}
$$

It is evident that $\lim _{M \rightarrow \infty} S(2 p M)$ is bounded provided that each $\lim _{M \rightarrow \infty} S_{i}(2 p M)$ is bounded.

The mean value theorem gives the following upper and lower bounds on $S_{i}(2 p M)$ :

$$
\sum_{m=1}^{M} f^{(D+i)}(2 p m-2 m+1), \quad \sum_{m=1}^{M} f^{(D+i)}(2 p m) .
$$

The choice of upper and lower depends on the sign of $f^{(D+i+1)}$. It now follows from the Maclaurin-Cauchy integral test for convergence [1, p. 294], [2, p. 33] that the bounding series both converge as $M \rightarrow \infty$ if and only if $\lim _{x \rightarrow \infty} f^{(D+i-1)}(x)$ is bounded. Now, if $\lim _{x \rightarrow \infty} f^{(D-1)}(x)$ is bounded, $\lim _{x \rightarrow \infty} f^{(D+i-1)}(x)=0$ for $i \geqslant 1$. It follows at once that the sole property of $[j]$ which determines the partial convergence of $[j] f$ is its differential order, $D$.

We have thus established the theorem. 
THEOREM 6.1. The alternating series [j] $f$ is partially convergent if and only if $\lim _{x \rightarrow \infty} f^{(D-1)}(x)$ is bounded, where $D$ is the differential order of the template $[j]$.

We then have

THEOREM 6.2. The alternating series $[j] f$ is convergent if and only if $\lim _{x \rightarrow \infty} f(x)=0$. Since $D \geqslant 1$, it follows from Theorem 6.1 that all series with

Since $D \geqslant 1$, it follows from Theorem 6.1 that all series with $\lim _{x \rightarrow \infty} f(x)=0$. are partially convergent; so, in view of Theorem 3.1, they are convergent. For $p=1$ this theorem is simply Leibnitz's rule $[1$, p. 131] that (in the present terminology) alternating series of degree 1 are convergent if and only if $f(x)$ decreases to zero.

Evidently, we have also

THEOREM 6.3. The alternating series $[j] f$ is divergent, if and only if $\lim _{x \rightarrow \infty} f^{(D-1)}(x)$ does not exist.

7. Examples of Partial Convergence. Tables 2 and 3 give some examples of the consequences of Theorems 6.1,6.2, and 6.3. Table 2 sets out the convergence, partial convergence, and divergence of $[j] f$ when $f(x)=(x+a)^{v+1 / 2}$ for $v=-2,-1,0,1,2$. The same table holds, of course, for any sequence of nonintegral powers $v+z$ with $0<z<1$.

TABLE 2

Partial convergence of alternating series of order $D$ for $f$ 's fractional power functions

\begin{tabular}{|c|c|c|c|c|c|}
\hline & & & D & & \\
\hline f & 0 & 1 & 2 & 3 & 4 \\
\hline$\left(x_{0}+x\right)^{-3 / 2}$ & CV & $\mathrm{CV}$ & CV & CV & CV \\
\hline$\left(x_{0}+x\right)^{-1 / 2}$ & DV & $c V, 3$ & $\mathrm{CV}, 2$ & $\mathrm{CV}, 1$ & CV \\
\hline$\left(x_{0}+x\right)^{1 / 2}$ & DV & DV & $\mathrm{PC}, 2$ & $\mathrm{PC}, 1$ & PC \\
\hline$\left(x_{0}+x\right)^{3 / 2}$ & DV & DV & DV & $\mathrm{PC}, 1$ & PC \\
\hline$\left(x_{0}+x\right)^{5 / 2}$ & DV & DV & DV & DV & PC \\
\hline
\end{tabular}

Notes: 1. Results for nonalternating series are shown in column $D=0$.

2. $x_{0}>-1$.

3. $\mathrm{CV}$, convergent. $\mathrm{PC}$, partly convergent. DV, divergent.

4. A numeral following CV or PC indicates the number of sums for these cases given for $x_{0}=10$ in Table 4 . 
Table 3 gives similar information for certain $f$ 's on the margins of convergence $[2$, p. 35], [3, p. 43].

TABLE 3

Partial convergence of alternating series of order $D$ for $f$ 's on the margins of convergence

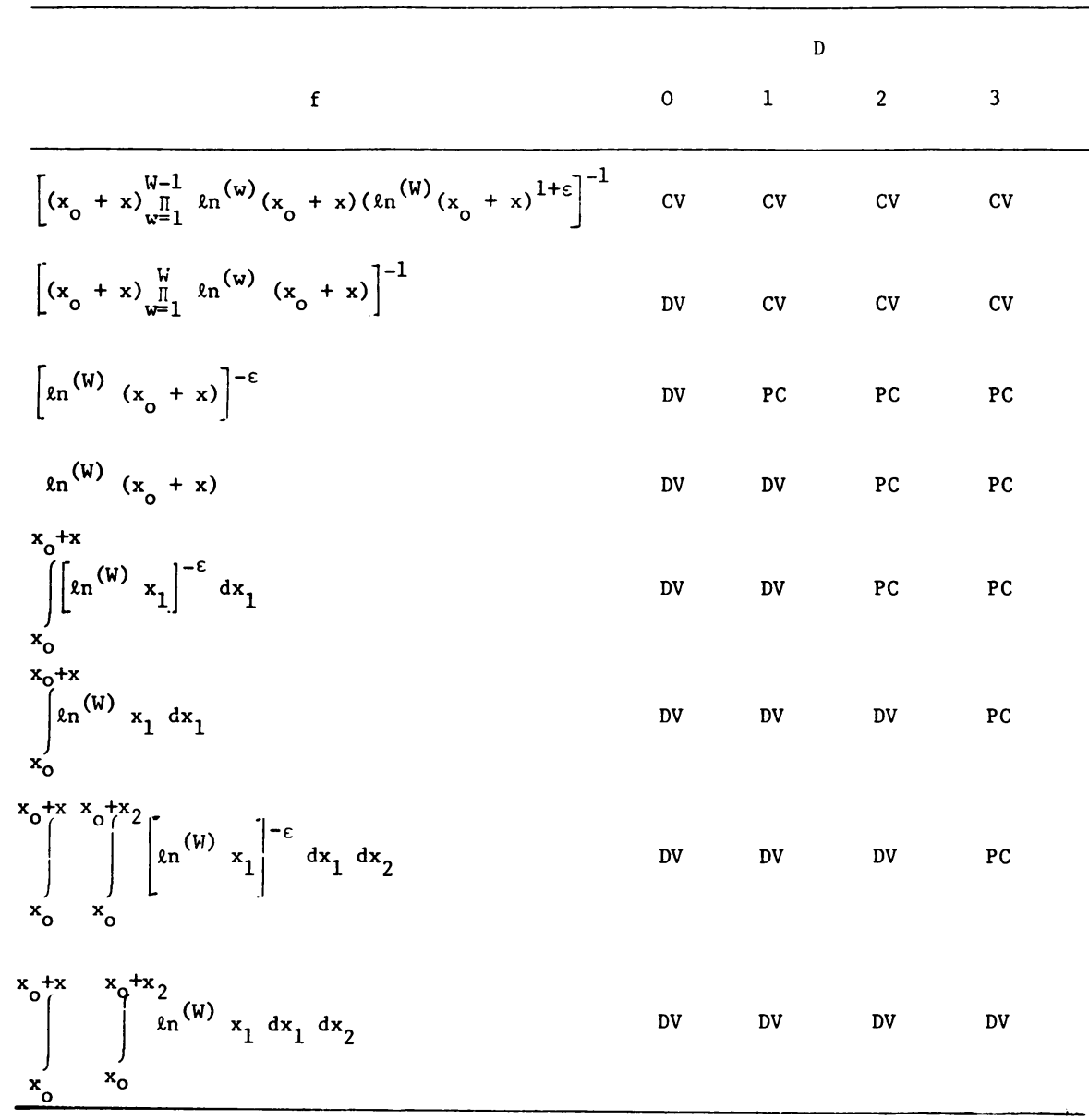

Notes: 1. Results for nonalternating series are given in the column $D=0$.

2. Everywhere $x_{0}$ is taken large enough to avoid negative logarithms.

3. $\ln ^{(w)} x$ denotes the $w$-fold repeated logarithm of $x$. Thus $\ln ^{(2)} x$ means $\ln \ln x$.

4. $W$ is finite, but may be taken as large as we please. $\epsilon$ is definitely positive nonzero, but may be taken as small as we please.

5. CV, convergent. PC, partly convergent. DV, divergent.

8. The Improved Euler-Maclaurin Expansion. We sum the alternating series which interest us here by means of the following improved form of the EulerMaclaurin expansion [4]:

$$
\sum_{n=1}^{N} f\left(x_{0}+n h\right) \sim h^{-1} \int_{x+1 / 2 h}^{x_{0}+(N+1 / 2) h} f(x) d x
$$

$$
+\sum_{r=1}^{\infty} a_{r} h^{2 r-1}\left[f^{(2 r-1)}\left(x_{0}+1 / 2 h\right)-f^{(2 r-1)}\left(x_{0}+(N+1 / 2) h\right)\right]
$$


with

$$
a_{r}=\frac{1-2^{1-2 r}}{(2 r !)} B_{2 r}=-\frac{B_{2 r}(1 / 2)}{(2 r !)}
$$

The $B_{2 r}$ are the Bernoulli numbers [2]. Values of $a_{r}$ for $r=0$ to 6 are tabulated in [4]. $a_{0}=-1 ; a_{1}=1 / 24 ; a_{2}=-7 / 5,760 ; a_{3}=31 / 967,680$.

A compact result follows from (8) when the series $[j] f$ is partially convergent and the template $[j]$ is pure. Putting (5) into (1), and then using (8), yields in the limit as $M \rightarrow \infty$,

$$
S^{(p)} \sim \frac{\alpha_{0}}{2 p} \sum_{r=0}^{\infty}(2 p)^{2 r} a_{r} f^{(2 r+D-1)}\left(\theta_{r}\right), \quad 1-p \leqslant \theta_{r} \leqslant p .
$$

We write $S^{(p)}$ for $\lim _{M \rightarrow \infty}(2 p M)$.

For a convergent alternating series with the pure template $[+-], p=1, D=1$, $\alpha_{0}=-1$, and (10) reduces to

$$
S \sim-1 / 2 \sum_{r=0}^{\infty} 2^{2 r} a_{r} f^{(2 r)}\left(\theta_{r}\right), \quad 0 \leqslant \theta_{r} \leqslant 1
$$

A simple estimate of $S^{(p)}$ when [j] is pure follows from (10). Retaining only the leading term of the expansion, replacing $\theta_{0}$ by its midrange value, namely $1 / 2$, and using (6), we obtain

$$
S_{*}^{(p)}=-\frac{\mu(D) f^{(D-1)}(1 / 2)}{2 p(D !)}
$$

where we write $S_{*}^{(p)}$ for this estimate of $S^{(p)}$. When the series is convergent we replace $S_{*}^{(p)}$ in (12) by $S_{*}$, since it is then an estimate of $S$. In Section 10 the efficiency of (12) is tested by comparing $S_{*}$ or $S_{*}^{(p)}$ with more precise estimates of $S$ or $S^{(p)}$ found using (8).

9. Euler's Transformation. The classical technique for summing slowly convergent alternating series of degree 1 is Euler's transformation [1, p. 144], [2, p. 62], [5, Eq. 3.6.27]. On the other hand, the improved Euler-Maclaurin expansion (8) has been found superior to Euler's transformation when applied to the practical summation of series of this type [4]. Using the mean value theorem, we may put Euler's transformation into a form comparable to (11):

$$
S=1 / 2 \sum_{r=0}^{\infty}(-1 / 2)^{r} f^{(r)}\left(\phi_{r}\right), \quad 1 \leqslant \phi_{r} \leqslant 1+r
$$

We see that the decreasing terms leading off the improved Euler-Maclaurin expansion (11) tend to decrease more rapidly than those of (13), at least so long as the nearest singularity of $f$ is not very much closer to the points $x=\theta_{r}$ than to the points $x=\phi_{r}$. The superiority of (8) for practical summation is therefore unsurprising. 
10. Practical Summation of Convergent and Partially Convergent Series. For the practical arithmetic summation of convergent or partially convergent alternating series of degree $p$ we use (8) in the following form:

$$
S \text { or } S^{(p)} \sim \frac{1}{2 p} \sum_{r=0}^{\infty}(2 p)^{2 r} a_{r} \sum_{q=1}^{2 p} j_{q} F^{(2 r)}(q-p), \quad F(x)=\int f(x) d x
$$

For $r>1$ successive terms of asymptotic expansion (14) alternate in sign so that upper and lower bounds on $S$ or $S^{(p)}$ are readily established. If greater accuracy is required when only the first few terms decrease in magnitude, this can be achieved by arithmetically summing leading terms of $[j] f$, and applying (14) to the series that remains.

TABLE 4

Sums of alternating series $[j]$ f of order $D$

\begin{tabular}{|c|c|c|c|c|c|c|c|c|}
\hline $\mathbf{f}$ & [j] & D & $\begin{array}{l}\text { Cv } \\
\text { or } \\
\text { PC }\end{array}$ & $r=1$ & $s_{(r)}$ or $s_{(r)}^{(p)}$ & 3 & $\begin{array}{l}S^{\star} \\
\text { or } \\
s s_{k}(p)\end{array}$ & $\begin{array}{c}s_{\star} / s \\
s_{\star}^{(p)} / s^{g r}(p)\end{array}$ \\
\hline \multirow[t]{6}{*}{$(10+x)^{-1 / 2}$} & +- & 1 & $\mathrm{cv}$ & 0.15417160 & 0.15417324 & 0.15417320 & 0.1543 & 1.001 \\
\hline & ++-- & 1 & $\mathrm{Cv}$ & 0.307792 & 0.307847 & 0.307841 & 0.3086 & 1.002 \\
\hline & +++-- & 1 & cv & 0.46018 & 0.46064 & 0.46053 & 0.4629 & 1.005 \\
\hline & +--+ & 2 & cv & 0.007229 & 0.007241 & 0.007239 & 0.00734 & 1.014 \\
\hline & $++\cdots+++$ & 2 & $\mathrm{cv}$ & 0.0273 & $0.0283^{\star}$ & 0.0276 & 0.0294 & 1.04 \\
\hline & +--+-++ & 3 & $\mathrm{cv}$ & 0.00171 & $0.00199^{\circ}$ & 0.00172 & 0.00210 & 1.06 \\
\hline \multirow[t]{3}{*}{$(10+x)^{1 / 2}$} & +-+ & 2 & PC & -0.076906 & -0.076919 & -0.076918 & -0.0772 & 1.004 \\
\hline & ++---++ & 2 & PC & -0.3076 & $-0.3087^{\star}$ & -0.3083 & -0.309 & 1.001 \\
\hline & +-++++ & 3 & PC & -0.0072 & $-0.0074^{\star}$ & -0.0073 & -0.0073 & 0.99 \\
\hline$(10+x)^{3 / 2}$ & +-++++ & 3 & PC & 0.2284 & $0.2291^{*}$ & 0.2288 & 0.231 & 1.008 \\
\hline
\end{tabular}

Notes: 1. CV, convergent. PC, partly convergent.

2. For convergent series, $S_{(r)}$ is estimate of $S$ given by $r+1$ terms of expansion (5). For partly convergent series, $s(p)$ is estimate of $s^{(p)}$ given by $r+1$ terms of expansion (5).

3. An asterisk indicates a sum to the term before the numerically least. In some other cases for $r=2$ or 3 further calculations are needed to identify the numerically least term unequivocally.

4. $S_{*}$ and $S_{*}^{(p)}$ are estimates of $S$ and $S^{(p)}$ given by (12).

Table 4 shows for 10 alternating series the estimates of $S$ or $S^{(p)}$ found by summing (14) to $r=1,2$, and 3. For all the series summation to $r=2$ (i.e. a 3-term sum) gives usefully close upper and lower bounds on $S$ or $S^{(p)}$.

All the templates of Table 4 are pure, and it is of interest to compare the modified Euler-Maclaurin sums obtained from (14) with $S_{*}$ or $S_{*}^{(p)}$, the estimates from the simple formula (12). We see that (12) always gives the order of magnitude of the sum correctly. The error is $1 \%$ or less for 7 series, and is less than $6 \%$ for all 10 . Unsurprisingly, formula (12) is least accurate when expansion (14) is least accurate. 
11. The Frequency of Partial Convergence. When, for an antisymmetric template, we have $\lim _{x \rightarrow \infty} f^{(D-1)}(x)=0$, the sum of $p M$ terms converges as well as that of $2 p M$ terms, with $S^{(1 / 2 p)}=S^{(p)}$. More elaborate patterns of partial convergence evidently occur for templates with higher orders of antisymmetry. The character of the relevant theorems is clear, but we shall not develop them here. We observe in conclusion that the four partly convergent series of Table 4 converge for sums of $2 M, 4 M$, $2 M$, and $4 M$ terms, even though they are of order $2,4,4$, and 4 , respectively.

12. Summing Finite Series. With $S^{(p)}$ known, $S(N)$ for $N$ large but finite can be estimated simply. We take as an illustration the series $[+--+-++-](10+x)^{3 / 2}$ for $N$ taking all integral values in $10^{6} \pm 4$. To two decimals $S\left(10^{6}\right)=S^{(8)}=0.23$, the numerical value following from Table 4. Neighboring $S(N)$ 's are then found by simple addition. The resulting values are shown in Table 5. The table illustrates well the fluctuating character of the sums of partially convergent series. Note that here every fourth sum converges even though $p=4$.

\section{TABLE 5}

\begin{tabular}{lr}
$\begin{array}{r}\text { Values of } S\left(+--+++-,(10+x)^{3 / 2}, N\right) \\
\text { for } 10^{6}-4 \leqslant N \leqslant 10^{6}+4\end{array}$ \\
\hline $\mathrm{N}$ & \multicolumn{1}{c}{$\mathrm{S}(\mathrm{N})$} \\
\hline $10^{6}-4$ & 0.23 \\
$10^{6}-3$ & $-1,000,011,999.79$ \\
$10^{6}-2$ & $1,500.24$ \\
$10^{6}-1$ & 0.23 \\
$10^{6}$ & $1,000,016,500.28$ \\
$10^{6}+1$ & $-1,499.78$ \\
$10^{6}+2$ & 0.23 \\
$10^{6}+3$ & $-1,000,022,499.85$ \\
$10^{6}+4$ &
\end{tabular}

13. Concluding Remarks. We conclude with some brief remarks on generalizations and extension of the present work.

(a) Our approach can be extended from binary templates to ternary ones with the $j_{n}$ assuming the values $+1,0,-1$. Extension to templates with the $j_{n}$ taking any finite number of values (not necessarily including \pm 1 and 0 ) is possible; but series generated by such elaborate templates tend to be artificial and uninteresting.

(b) We may generalize also by relaxing the constraint that $\Sigma_{q=1}^{2 p} j_{q} \equiv 0$, but this seems unlikely to be particularly useful or illuminating. 
(c) There is an inverse strategy for generating partially convergent alternating series: For any given template $[j]$, we may write

$$
s(2 p m)=\sum_{q=1}^{2 p} j_{q} f(2 p(m-1)+q),
$$

where $s$ is the sum of the $m$ th group of $2 p$ terms. We now choose the function $s(2 p m)$ so that the series $\Sigma_{m=1}^{\infty} s(2 p m)$ is convergent and has the sum $T$. We then have that, for any solution $\Phi(x)$ of the difference equation

$$
s(x)=\sum_{q=1}^{2 p} j_{q} f(x-2 p+q),
$$

the series $[j] \Phi$ is partially convergent with $S^{(p)}=T$.

CSIRO Division of Environmental Mechanics

P. O. Box 821

Canberra City, ACT 2601, Australia

1. K. KNOPP, Theory and Application of Infinite Series, 2nd ed., Blackie, London, 1951.

2. T. J. I'A. BROMWICH, An Introduction to the Theory of Infinite Series, 2nd ed., Macmillan, London, 1926.

3. G. H. HARDY, Orders of Infinity, 2nd ed., Cambridge Univ. Press, Cambridge, 1924.

4. J. R. PHILIP, "The symmetrical Euler-Maclaurin summation formula," The Mathematical Scientist. (In press.)

5. M. ABRAMOWITZ, "3. Elementary analytical methods," in Handbook of Mathematical Functions (M. Abramowitz \& I. A. Stegun, Eds.), Nat. Bur. Standards Appl. Math. Series 55, U. S. Dept. of Commerce, Washington, D. C., 1964. MR 29 \#4914. 